\title{
Improved automated lattice perturbation theory in background field gauge
}

\section{T.C. Hammant, R.R. Horgan, C.J. Monahan}

DAMTP, CMS, University of Cambridge, Cambridge CB3 OWA, U.K.

\section{A.G. Hart*}

Cray Exascale Research Initiative, JCMB, King's Buildings, Edinburgh EH9 3JZ, U.K.

\section{E.H. Müller}

SUPA, School of Physics, University of Edinburgh, Edinburgh EH9 3JZ, U.K.

\section{A. Gray, K. Sivalingham}

EPCC, JCMB, King's Buildings, Edinburgh EH9 3JZ, U.K.

\section{G.M. von Hippel}

Institut für Kernphysik, Johannes-Gutenberg-Universität Mainz, 55099 Mainz, Germany

hippel@kph.uni-mainz.de

\begin{abstract}
We present an algorithm to automatically derive Feynman rules for lattice perturbation theory in background field gauge. Vertices with an arbitrary number of both background and quantum legs can be derived automatically from both gluonic and fermionic actions. The algorithm is a generalisation of our earlier algorithm based on prior work by Lüscher and Weisz. We also present techniques allowing for the parallelisation of the evaluation of the often rather complex lattice Feynman rules that should allow for efficient implementation on GPUs, but also give a significant speed-up when calculating the derivatives of Feynman diagrams with respect to external momenta.
\end{abstract}

The XXVIII International Symposium on Lattice Field Theory, Lattice2010

June 14-19, 2010

Villasimius, Italy

\footnotetext{
* previous address: SUPA, School of Physics, University of Edinburgh, Edinburgh EH9 3JZ, U.K.

${ }^{\dagger}$ new address: Meteorological Office, FitzRoy Road, Exeter, Devon EX1 3PB, U.K.

$\ddagger$ Speaker.
} 


\section{Automated Lattice Perturbation Theory}

While the lattice offers a fully nonperturbative regulator for Quantum Field Theory, perturbative calculations still play an important role in renormalising, matching and improving operators, both those appearing in the action and those used to measure observables. For the highly improved actions now widely used, such as the Highly Improved Staggered Quark (HISQ) action [1] or moving NRQCD, [2] manual derivation and implementation of the Feynman rules would be highly impractical. Automated methods are therefore required. A general algorithm to derive the Feynman rules for arbitrary traced closed Wilson loops was derived by Lüscher and Weisz in [3]. We have generalised this algorithm to include fermionic fields [4] and have implemented it in the HiPPy/HPsrc packages [5].

\subsection{The HiPPy package}

HiPPy is an is an automated tool for generating Feynman rules from arbitrary lattice actions, which is written entirely in Python [6]. Starting from the perturbative expansion $U_{\mu}(\boldsymbol{x})=\mathrm{e}^{g_{0} A_{\mu}\left(\boldsymbol{x}+\frac{\mu}{2}\right)}$ of the link variables, the action is expanded as

$$
\begin{aligned}
\mathscr{L} & =\operatorname{Tr}\left(U_{\mathscr{C}}\right)+\bar{\psi} \Gamma U_{\mathscr{D}} \psi \\
& =\sum_{r} \frac{g_{0}^{r}}{r !} V_{\mu_{1} \cdots \mu_{r}}^{a_{1} \cdots a_{r}} A_{\mu_{1}}^{a_{1}} \cdots A_{\mu_{r}}^{a_{r}}+\sum_{r} \frac{g_{0}^{r}}{r !} \bar{\psi}^{b} \tilde{V}_{\mu_{1} \cdots \mu_{r}}^{a_{1} \cdots a_{r}, b c} A_{\mu_{1}}^{a_{1}} \cdots A_{\mu_{r}}^{a_{r}} \psi^{c}
\end{aligned}
$$

with vertex functions

$$
\begin{aligned}
V_{\mu_{1} \cdots \mu_{r}}^{a_{1} \cdots a_{r}}\left(k_{1}, \ldots, k_{r}\right) & =\operatorname{Tr}\left(t^{a_{1}} \cdots t^{a_{r}}\right) \times \sum_{i} f_{i} \mathrm{e}^{i \sum_{j} k_{j} \cdot v_{i, j}} \\
\tilde{V}_{\mu_{1} \cdots \mu_{r}}^{a_{1} \cdots a_{r}, b c}\left(k_{1}, \ldots, k_{r} ; p, q\right) & =\left(t^{a_{1}} \cdots t^{a_{r}}\right)_{b c} \times \sum_{i} f_{i} \Gamma_{\alpha_{i}} \mathrm{e}^{i\left(p \cdot x_{i}+q \cdot y_{i}+\sum_{j} k_{j} \cdot v_{i, j}\right)}
\end{aligned}
$$

giving the Feynman rules.

The algorithm for achieving this expansion starts from the encoding of individual terms in the vertex function as so-called entities

$$
E_{i}=\left(\boldsymbol{x}_{i}, \boldsymbol{y}_{i} ; \boldsymbol{v}_{i, 1}, \ldots, \boldsymbol{v}_{i, r} ; \alpha_{i}\right)
$$

each of which carries an amplitude $f_{i}$. The crucial property of entities is the multiplication rule

$$
E E^{\prime}=\left(\boldsymbol{x}, \boldsymbol{y}^{\prime}+\boldsymbol{c} ; \boldsymbol{v}_{1}, \ldots, \boldsymbol{v}_{r}, \boldsymbol{v}_{1}^{\prime}+\boldsymbol{c}, \ldots, \boldsymbol{v}_{s}^{\prime}+\boldsymbol{c} ; \alpha_{k}\right)
$$

where $\boldsymbol{c}=\boldsymbol{x}^{\prime}-\boldsymbol{y}$ and $\alpha_{k}$ is defined via $\Gamma_{\alpha_{i}} \Gamma_{\alpha_{j}}=\phi_{\alpha_{i} \alpha_{j}} \Gamma_{\alpha_{k}}$. Entities differing by only translations are equivalent by momentum conservation. Additional structure (e.g. a non-trivial colour structure) can also be encoded by adding additional fields to the entity and amending the entity algebra accordingly.

A field is then defined as a double mapping

$$
F=\left\{\left(\mu_{1}, \ldots, \mu_{r}\right) \mapsto\left\{E_{i} \mapsto f_{i}\right\}\right\}
$$


which encodes a generic Wilson line, and multiplication of field objects is defined accordingly in terms of entity multiplication by

$$
F F^{\prime}=\left\{\left(\mu_{1}, \ldots, \mu_{r}, \mu_{1}^{\prime}, \ldots, \mu_{s}^{\prime}\right) \mapsto\left\{E E^{\prime} \mapsto C_{r s} \phi f f^{\prime}\right\}\right\}
$$

where $C_{r s}=(r+s) ! /(r ! s !)$ is a combinatorial factor and $\phi$ is the phase from the multiplication of the spin matrices belonging to the entities $E$ and $E^{\prime}$, as defined above. Addition of field objects is defined by the addition of the amplitudes belonging to the individual entities, with the amplitude in the sum of an entity present in only one of the summands being set to its amplitude in that summand.

The basic building block from which smeared links, operators and actions are constructed in an iterative fashion is the simple link encoded as

$$
U_{\mu}(\boldsymbol{x})=\mathrm{e}^{g_{0} A_{\mu}\left(\boldsymbol{x}+\frac{\hat{\mu}}{2}\right)}=\left\{(\mu, \ldots, \mu) \mapsto\left\{\left(\mathbf{0}, \hat{\mu} ; \frac{\hat{\mu}}{2}, \ldots, \frac{\hat{\mu}}{2} ; 0\right) \mapsto 1\right\}\right\}
$$

and predefined building blocks (e.g. smeared links, covariant derivatives and field strength tensors) constructed from this are provided as part of HiPPy.

\subsection{The HPsrc package}

The HPsrc package consists of a suite of Fortran 95 modules complementing HiPPy. While the output of HiPPy is in principle suitable to being converted directly into an analytic form, this is not usually necessary or even useful in lattice perturbation theory. We therefore have implemented routines that read in the HiPPy-generated Feynman rules at runtime and use them to construct the vertex functions and propagators for given momenta on the fly. This also offers the great advantage of being able to write Feynman diagrams in an action-blind way, so as to be able to recompute the same quantities for another action without needing to write new code. HPsrc also provides facilities for automated differentiation of Feynman diagrams, so that neither analytic manipulations nor inaccurate numerical derivatives are needed.

\section{Incorporating Background Fields}

The background field technique has long been known as a valuable tool in field theory. In [7], Lüscher and Weisz showed that the theorem about dimensional regularisation stating that renormalisation of the effective action does not require additional counterterms beyond those needed for the renormalisation of the action holds also in the case of lattice gauge theory. This makes it possible to use the background field technique to perform calculations such as relating the bare lattice coupling to the $\overline{\mathrm{MS}}$ coupling [8]. To determine the coefficient of the $\boldsymbol{\sigma} \cdot \boldsymbol{B}$ term in the (moving) NRQCD action [9], only the background field technique can guarantee the gauge invariance of higher-dimensional operators which will necessarily be generated in an effective theory such as (m)NRQCD. This makes it desirable to incorporate support for the background field method into the HiPPy/HPsrc packages. 


\subsection{Background fields in HiPPy}

We decompose our fields into a background field $B_{\mu}$ and quantum fluctuations $q_{\mu}$ by parametrising the basic gauge link as

$$
U_{\mu}(\boldsymbol{x})=\mathrm{e}^{g_{0} q_{\mu}\left(\boldsymbol{x}+\frac{a}{2}\right)} \mathrm{e}^{B_{\mu}\left(\boldsymbol{x}+\frac{a}{2}\right)}
$$

We note that this does not affect the combinations of $\boldsymbol{x}, \boldsymbol{y}, \boldsymbol{v}_{i, j}$ that are possible, and that hence the entity algebra remains unaffected. However, the gluon fields living at each lattice point $v$ can now be either quantum or background, with the quantum fields always appearing to the left of the background fields coming from the same link, and thus the field objects need to keep track of nature of individual gluon fields.

This leads to a new mapping structure for field objects given now by

$$
F=\left\{\left(\mu_{1}, \ldots, \mu_{r}\right) \mapsto\left\{E_{i} \mapsto\left(f_{i}^{q \cdots q}, \ldots, f_{i}^{B \cdots B}\right)\right\}\right\}
$$

with an order- $r$ entity being mapped to an $2^{r}$-tuple of amplitudes.

With this structure, multiplication of fields now assigns

$$
f_{k}^{x_{1} \cdots x_{r} y_{1} \cdots y_{s}}:=C_{r s} \phi f_{i}^{x_{1} \cdots x_{r}} f_{j}^{y_{1} \cdots y_{s}}
$$

where $C_{r s}$ and $\phi$ are defined as before, and the simple link becomes

$$
U_{\mu}(\boldsymbol{x})=\mathrm{e}^{g_{0} A_{\mu}\left(\boldsymbol{x}+\frac{\hat{\mu}}{2}\right)}=\left\{(\mu, \ldots, \mu) \mapsto\left\{\left(\mathbf{0}, \hat{\mu} ; \frac{\hat{\mu}}{2}, \ldots, \frac{\hat{\mu}}{2} ; 0\right) \mapsto\left(1, \ldots, f^{q \cdots q B \cdots B}, \ldots, 1\right)\right\}\right\},
$$

where

$$
f^{q^{s} B^{r-s}}=\frac{r !}{s !(r-s) !}
$$

and all other $f^{x}$ vanish identically. This definition maintains the ordering of the background and quantum fields throughout the expansion procedure. We note that this precludes performing any symmetrisation over the gluon fields at this stage, and that in background gauge all symmetrisation is to be deferred to the evaluation of the Feynman diagrams.

\subsection{Background field gauge in HPsrc}

In order to support background field calculations, we also need to extend HPsrc so that it supports fixing to background field gauge.

The gauge fixing term for this is

$$
\mathscr{L}_{g f}=-\frac{1}{2 \xi} \operatorname{Tr}\left(D_{\mu}^{*} q_{\mu}\right)^{2}
$$

with

$$
D_{\mu}^{*} f(x)=\left[f(x)-\mathrm{e}^{-B_{\mu}\left(x-\frac{\hat{\mu}}{2}\right)} f(x-\hat{\mu}) \mathrm{e}^{B_{\mu}\left(x-\frac{\hat{\mu}}{2}\right)}\right],
$$

which gives rise to additional terms in all purely gluonic vertices with exactly two quantum fields. These terms have been implemented in HPsrc for the propagator and three-gluon vertex.

Similarly, the Fadeev-Popov ghost action in background field gauge involves background covariant derivatives $D_{\mu}$ instead of finite differences, and $\operatorname{Ad}\left(q_{\mu}\right)$ instead of $\operatorname{Ad}\left(A_{\mu}\right)$. 


\section{Accelerated Evaluation of Feynman Diagrams}

\subsection{Accelerated Automatic Differentiation}

In HPsrc, we use TaylUR [10] for the automatic computation of derivatives with respect to an external momentum $\boldsymbol{P}$. In this way, derivative information is automatically propagated from vertices and propagators to Feynman diagrams and quantities constructed therefrom, but in order to save computation time, the derivatives of the vertex functions themselves are constructed explicitly in the HPsrc code.

For a momentum decomposed as $\boldsymbol{k}_{j}=\overline{\boldsymbol{k}}_{j}+r_{j} \boldsymbol{P}$, the derivative of the reduced vertex reads

$$
\partial_{P_{1}}^{n_{1}} \cdots \partial_{P_{D}}^{n_{D}} Y=\sum_{i} f_{i} \prod_{\mu=1}^{D}\left(i \sum_{j} r_{j} v_{i, j, \mu}\right)^{n_{\mu}} \mathrm{e}^{i \sum_{j} k_{j} \cdot v_{i, j}}
$$

We note that the $\Pi(\ldots)$ term above is momentum-independent, and needs to be computed only once in order to compute the derivatives for each momentum in a set of momenta with identical routings $r_{j}$; only the exponential needs to be recomputed for each momentum. It is therefore possible to achieve a significant speed-up by momentum vectorisation, such that the vertex function is called with a vector of momenta with identical routings $r_{j}$ and returns a vector of values.

\section{GPU acceleration of Reduced Vertices}

The main work (about 85\%) in a perturbative calculation using HPsrc comes from evaluating the reduced vertex. This function is particularly suitable for parallelisation using a General Purpose GPU (GPGPU). Data transfer times are small and the momentum vectorisation discussed above makes the overhead per momentum point negligible (as the monomial data can be reused).

The reduced vertex routine was extracted as a separate kernel for testing. Derivatives of the reduced vertex were not initially calculated. The kernel consists of a two-level loop nest. The outer loop (over independent momentum points with loop index $n$ ) is trivially parallel. The inner loop (the sum over monomials with loop index $i$ ) contained some dependencies. We fixed the number of monomials at 8000 (representative of the HISQ action) and varied the number of points.

The kernel was accelerated on an NVIDIA Fermi C2050 GPU in two ways. First, a corresponding CUDA kernel was written and called via a C wrapper. Secondly, the original Fortran90 was accelerated using the directives implemented in the PGI compiler.

Initially, only the outer loop was parallelised, explicitly in the CUDA and automatically by the PGI compiler. In both cases, GPU performance was significantly increased by reordering the $k$ array so that index $n$ was the fastest-varying in memory. This allowed "coalescing" of loads from the GPU global memory.

For a wide range of problem sizes, the CUDA kernel (including data transfers) was around 52 times faster than the best version of the kernel running on a single core of an Intel Nehalem processor. The PGI directive version was only $20 \%$ slower than the CUDA kernel.

Further performance gains were obtained by restructuring the kernel so that loops over both $n$ and $i$ could be parallelised. When this was done in the PGI directive-accelerated kernel, the performance matched that of the original CUDA version. The same refactoring in the CUDA is currently in progress. Our results are summarised in Fig. 1. 


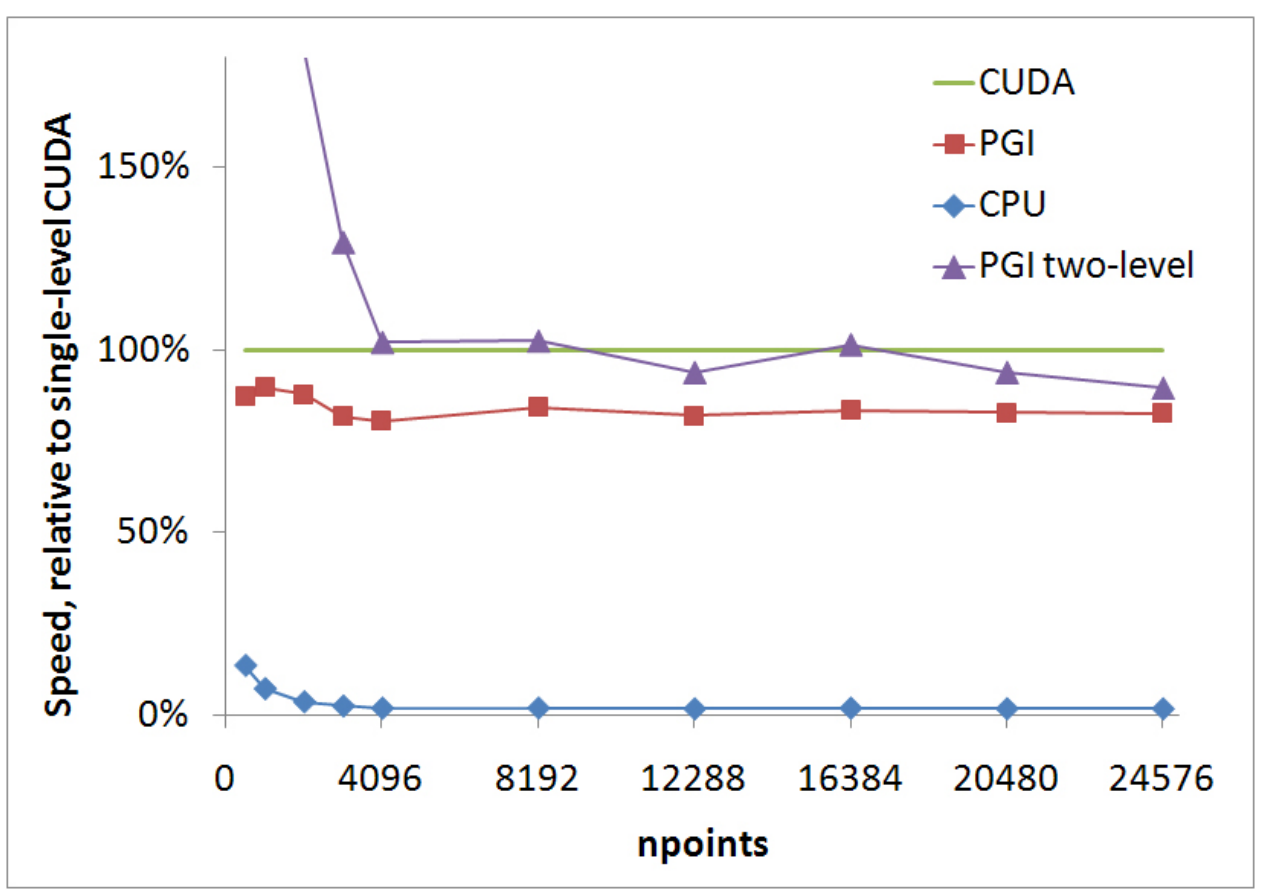

Figure 1: Comparing the performance of the HPsrc kernel on an NVIDIA Fermi GPU for increasing numbers of momentum points.

This work is now being ported into the main application.

\section{Summary}

The HiPPy/HPsrc package has been extended to enable calculations in background field gauge. The new functionality will be used to calculate to $\mathscr{O}\left(\alpha_{s}\right)$ corrections to the coefficients of the (m)NRQCD action.

The re-use of common routing information enables a significant speed-up of calculations involving automatic differentiation of vertices. A further speed-up can be achieved by rewriting the generic vertex functions as CUDA kernels. An optimised implementation is in preparation.

\section{References}

[1] HPQCD, E. Follana et al., Highly Improved Staggered Quarks on the Lattice, with Applications to Charm Physics, Phys. Rev. D75, 054502 (2007) [hep-lat/0610092].

[2] HPQCD, R.R. Horgan et al., Moving NRQCD for heavy-to-light form factors on the lattice, Phys. Rev. D 80 (2009) 074505 [arXiv:0906.0945].

[3] M. Lüscher, P. Weisz, Efficient Numerical Techniques For Perturbative Lattice Gauge Theory Computations, Nucl. Phys. B266 (1986) 309.

[4] A. Hart, G.M. von Hippel, R.R. Horgan, L.C. Storoni, Automatically generating Feynman rules for improved lattice field theories, J. Comput. Phys. 209 (2005) 340 [hep-lat/0411026]. 
[5] A. Hart, G.M. von Hippel, R.R. Horgan, E.H. Müller, Automated generation of lattice QCD Feynman rules, Comput. Phys. Commun. 180 (2009) 2698 [arXiv:0904.0375].

[6] available at http://www.python.org/

[7] M. Lüscher and P. Weisz, Background field technique and renormalization in lattice gauge theory, Nucl. Phys. B 452 (1995) 213 [arXiv:hep-lat/9504006].

[8] M. Lüscher and P. Weisz, Computation of the relation between the bare lattice coupling and the MS coupling in $S U(N)$ gauge theories to two loops, Nucl. Phys. B 452 (1995) 234 [arXiv:hep-lat/9505011].

[9] R.R. Horgan et al., work in progress.

[10] G. M. von Hippel, TaylUR, an arbitrary-order automatic differentiation package for Fortran 95, Comput. Phys. Commun. 174569 (2006) [physics/0506222]; ibid. 181705 (2010) [arXiv:0910.5111]. 